\title{
Alignment and Calibration Procedures of the Necsa Neutron Strain Scanner
}

\author{
D. Marais ${ }^{1, a^{*}}$, A.M. Venter ${ }^{1, b}$ and V. Luzin ${ }^{2, c}$ \\ ${ }^{1}$ Research and Development Division, Necsa SOC Limited, Pretoria, South Africa \\ ${ }^{2}$ Australian Centre for Neutron Scattering, ANSTO, Lucas Heights, NSW, Australia \\ adeon.marais@necsa.co.za, bandrew.venter@necsa.co.za, 'vll@ansto.gov.au
}

Keywords: Neutron Diffraction Instrument, Strain Scanner, Alignment and Calibration

\begin{abstract}
The alignment and calibration of neutron strain instruments directly affect the reliability and accuracy of neutron stress experiments. Procedures for the purpose of instrument optimization and characterization were established at Necsa's angular dispersive neutron strain scanner but may also be applicable to other diffraction instruments. Attention is given to the following aspects: sample table center of rotation, horizontally focusable multi-wafer silicon single crystal monochromator, neutron area detector offset and the instrument gauge volume.
\end{abstract}

\section{Introduction}

The Materials Probe for Internal Strain Investigations (MPISI) neutron diffraction strain scanner [1] is situated at the SAFARI-1 research reactor of the South African Nuclear Energy Corporation (Necsa) SOC Limited and is depicted in Fig. 1. A commissioning phase followed after a recent major instrument upgrade to improve measurement accuracy and precision.

In order to maximize the available neutron flux at the sample position and reduce the error in the measured diffraction angle (and consequently in the $\mathrm{d}_{\mathrm{hkl}}$ and calculated stress value), all components contributing to the neutron optical path of the instrument must be aligned to as high an accuracy as practically achievable. Complete instrument alignment is generally an iterative process as the system comprises of a number of interdependent components.

\section{Center of rotation}

The instrument reference point is measurable and is defined as the intersection of the sample table's center of rotation $(\mathrm{CoR})$ axis with the diffraction plane. In order to determine the CoR, the linear $x$ and $y$-axes (motors sx and sy) of the sample table should be adjusted such that an accurately machined calibration pin is not be displaced by more than $10 \%$ of the applicable gauge volume dimension when

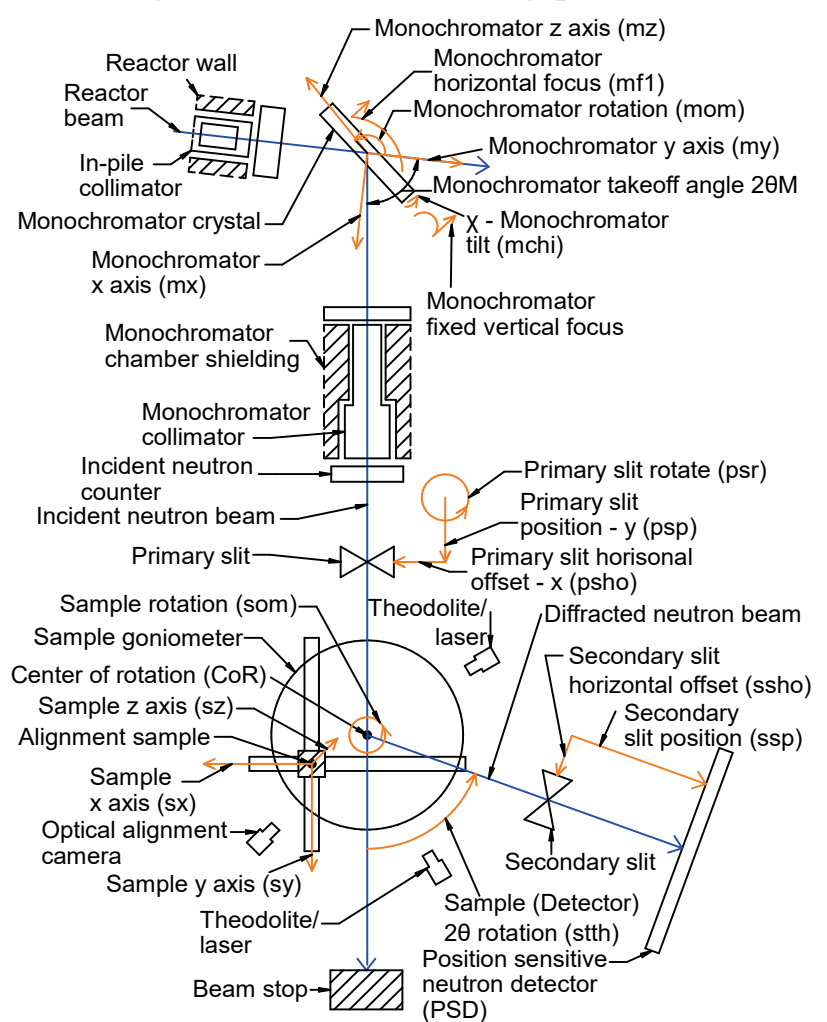

Fig. 1: Diagram of MPISI indicating selected motor names and components. 
driving the sample table's omega rotation axis (som motor) [2]. The pin location can be measured either using a digital dial gauge [3] or a telecentric camera alignment system [4]. Communication modules for both the digital dial gauge and the camera system were created in the MPISI control system SICS [5] in order to automate alignment procedures.

After the axes of the measurement device are aligned with the axes of the sample positioning table, the $x$ and $y$-axes offsets needed to move the alignment pin to the CoR must be determined. This can be done using an iterative procedure to solve a system of three equations [6], but this method is heavily dependent on starting the solution process with good initial values. In order to overcome this limitation, explicit equations (Eqs. 1 and 2) were developed to calculate the offsets by using four measurements taken $90^{\circ}$ apart (Fig. 2).

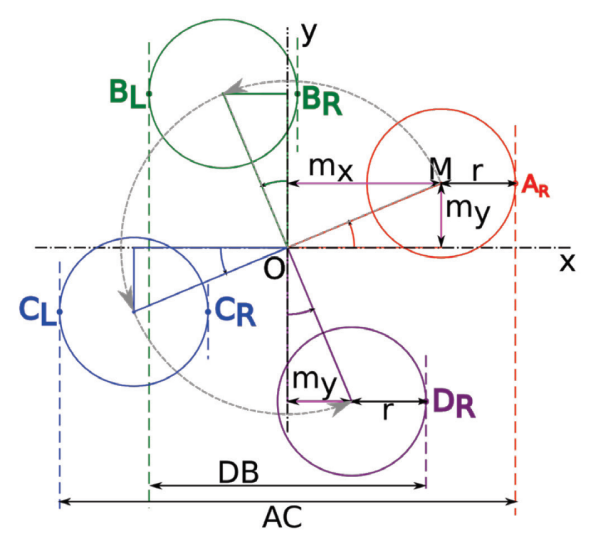

Fig. 2: Depiction of the displacement of a misaligned pin about the $C o R$ when rotated though $270^{\circ}$ at $90^{\circ}$ intervals.

$$
\begin{aligned}
& m_{x}=\frac{A_{R}-C_{R}}{2} . \\
& m_{y}=\frac{D_{R}-B_{R}}{2} .
\end{aligned}
$$

where:

$m_{x}=x$-axis offset

$m_{y}=y$-axis offset

$A_{R}=$ Gauge reading at position $\mathrm{A}\left(0^{\circ}\right)$

$B_{R}=$ Gauge reading at position $\mathrm{B}\left(90^{\circ}\right)$

$C_{R}=$ Gauge reading at position $\mathrm{C}\left(180^{\circ}\right)$

$D_{R}=$ Gauge reading at position $\mathrm{D}\left(270^{\circ}\right)$

Explicit equations (Eq. 3 and 4) can also be used to determine the offsets by using three measurements taken $45^{\circ}$ apart (Fig. 3). This method is very convenient when a large sample omega rotation is not possible such as when a mounted sample is restricting movement.

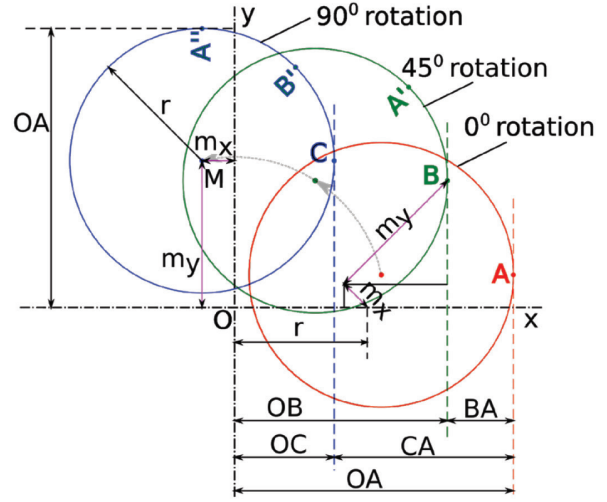

Fig. 3: Depiction of the displacement of a misaligned pin about the CoR when rotated though $90^{\circ}$ at $45^{\circ}$ intervals.

$$
\begin{aligned}
& m_{x}=\frac{1}{\sqrt{2}-1}\left(B A-C A+\frac{C A}{\sqrt{2}}\right) . \\
& m_{y}=C A-\frac{1}{\sqrt{2}-1}\left(B A-C A+\frac{C A}{\sqrt{2}}\right) .
\end{aligned}
$$

where:

$m_{x}=x$-axis offset

$m_{y}=y$-axis offset

$B A, C A=$ Measured distance (x-projection) between positions $\mathrm{B}$ and $\mathrm{A}$, and $\mathrm{C}$ and $\mathrm{A}$ respectively

Both methods were implemented on MPISI. The final pin position (relative) as measured with the digital dial gauge after using the camera alignment system is given in Fig. 4. When using the camera alignment system, the 3-measurement method should therefore not be used for gauge volume dimensions smaller than $0.5 \mathrm{~mm}$. 


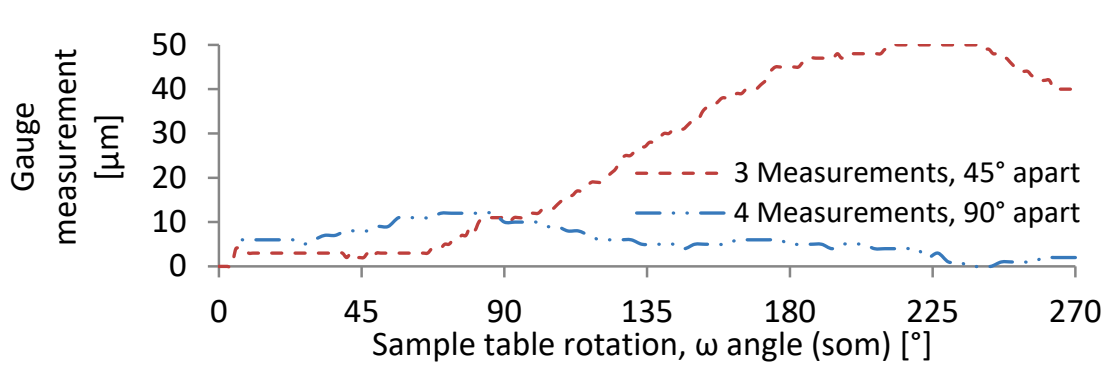

Fig. 4: Relative pin
position when
rotating the sample
table after two
different CoR
alignment
procedures.

\section{Monochromator alignment}

Ideally, the monochromator must be positioned such that the point where the monochromator assembly's center of rotation $\left(\mathrm{CoR}_{\mathrm{M}}\right)$ and tilt rotation center intersects is located at the intersection of the reactor beam and the required primary neutron beam. An approximation of the direction of the primary beam can be visually determined by replacing the monochromator collimator with a transparent Perspex plug that has a small hole ( $2 \mathrm{~mm}$ diameter $)$ drilled through its center. A laser is then directed through the hole into the monochromator chamber and the $x, y$, and $z$ offsets (corresponding to the $\mathrm{mx}$, my and $\mathrm{mz}$ motors) of the assembly are adjusted until the laser spot is observed on the center of the monochromator face. With reference to Fig. 6 , $x$ and $y$ are respectively tangential and parallel to the reactor beam with $z$ out of the page. The tilt axis (mchi motor) enables the monochromator to lean forward and backward.

With the Si (110) plane of the monochromator cut parallel to its face, the planes that are diffracted from when rotating the crystal in the horizontal plane about [110] within the constraints of the available space inside the monochromator chamber, are depicted in the stereographic projection given in Fig. 5. Si (110) was chosen due to a fixed monochromator takeoff angle through the monochromator chamber and space restrictions on the beam-port floor. By using this cut plane, appropriate reflections can be reached to obtain close to $90^{\circ}$ diffraction angles from typical engineering materials. Silicon has a cubic diamond crystal structure and therefore the angle $\varphi$ between two planes $\left(h_{1} k_{1} l_{1}\right)$ and $\left(h_{2} k_{2} l_{2}\right)$ is given by Eq. 5 [7]. Using this equation, the angles between diffracting planes were calculated and are depicted in Fig. 6 .

$$
\varphi=\cos ^{-1}\left(\frac{h_{1} h_{2}+k_{1} k_{2}+l_{1} l_{2}}{\sqrt{h_{1}^{2}+k_{1}^{2}+l_{1}^{2}} \sqrt{h_{2}^{2}+k_{2}^{2}+l_{2}^{2}}}\right) .
$$

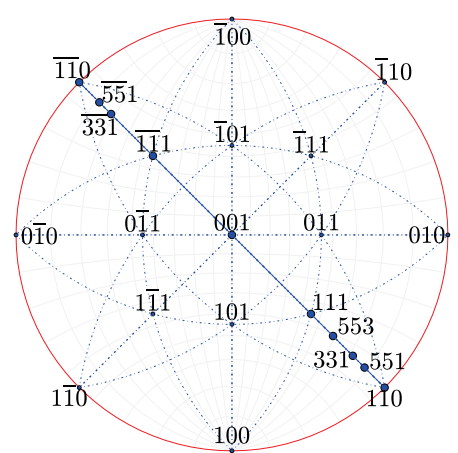

Fig. 5: Stereographic projection of a diamond cubic crystal structure showing poles relevant to MPISI's Si monochromator.

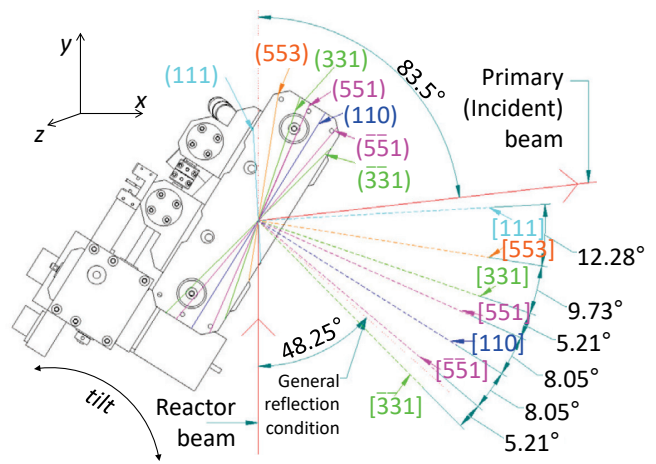

Fig. 6: Diagram of the MPISI Si crystal monochromator showing the orientation and direction of the crystal planes including the diffraction condition for a $83.5^{\circ}$ take-off angle. 
The monochromator rotation angle (mom motor) was step-scanned whilst recoding the neutron count rate provided by a neutron counter positioned in the primary beam. The resulting peaks (Fig. 7) were fit with Gaussian functions to determine the diffraction angles. The separation angles between peaks were then used to identify (index) the peaks. It should be noted that the structure factor for the (110) plane of $\mathrm{Si}$ is 0 , therefore the diffraction observed at the associated angle is primarily from the (220) reflection.

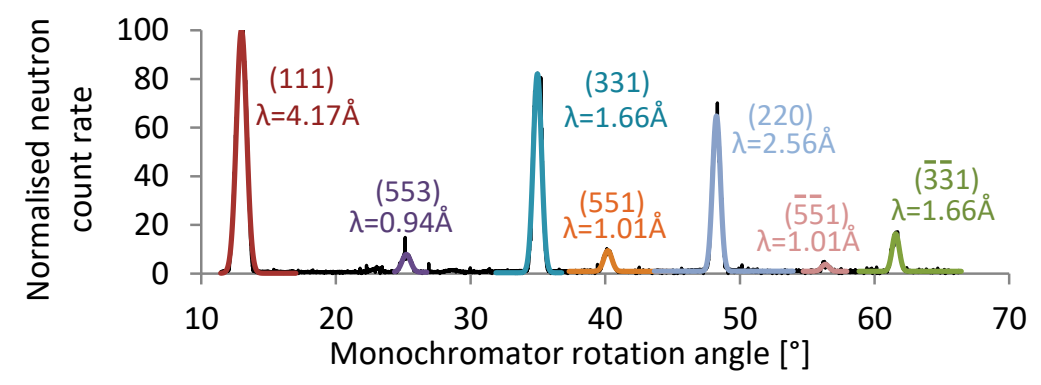

Fig. 7: Graph of the normalized neutron count rate at the monochromator exit port as a function of monochromator rotation angle. The primary wavelength for each peak is also given.

The (111) peak was selected to align the monochromator for maximum intensity. The monochromator $\mathrm{mx}$, my and mchi motors were sequentially step-scanned over the achievable translation ranges whilst recording the neutron count rate on the beam monitor. After each scan, a Gaussian function was fit to the acquired data in order to determine the motor position rendering maximum count rate.

A well-aligned monochromator not only delivers a high intensity neutron beam but also minimizes the spread in wavelength to the sample position. There is however a trade-off between observed integrated intensity and spread in wavelength which can be optimized using the figure of merit (FOM) [8] given in Eq. 6.

$$
F O M \propto \frac{\text { Intensity }}{F W H M^{2}}
$$

In order to optimize the FOM for the diffraction angle $\left(2 \theta_{\mathrm{S}}\right)$ range commonly used at MPISI, a mild steel pin was positioned on the CoR and the monochromator curvature (motor mf1) was step-scanned from $0.04 \mathrm{~m}^{-1}$ to $0.61 \mathrm{~m}^{-1}$ in 574 steps whilst recording the diffraction pattern on the neutron detector. The monochromator was positioned on the Si (331) reflection. Fig. 8a and b respectively show the Fe (211) diffraction peak and the resulting FOM as a function of horizontal monochromator curvature. The optimum curvature was obtained from the FOM by fitting a Gaussian function to the data.

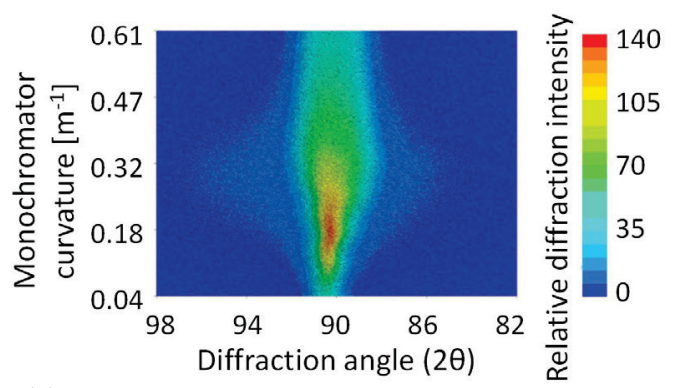

(a)

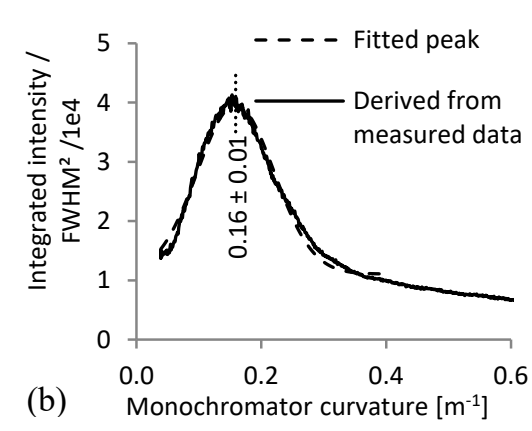

Fig. 8: Graphs of (a) the Fe (211) diffraction peak and (b) the resulting figure of merit, as a function of monochromator curvature.

The diffraction plane was aligned with the center of the detector by ensuring that the center of a low-angle diffraction cone coincides with the vertical center of the detector. To achieve this, a Mo (110) peak was measured at $43.8^{\circ} 2 \theta_{\mathrm{S}}$ using neutrons with a wavelength of $1.659 \AA$. The observed diffraction cone was then subdivided into 30 vertical sections (Fig. 9a) and each section 
integrated to produce a diffraction peak corresponding to a $y$-detector position. A Gaussian function was fit to each peak to determine the associated diffraction angles. These diffraction angles (as a function of $y$-detector position) was again fit with a Gaussian function to determine the center of the diffraction cone with respect to the detector position as is shown in Fig. 9.b.
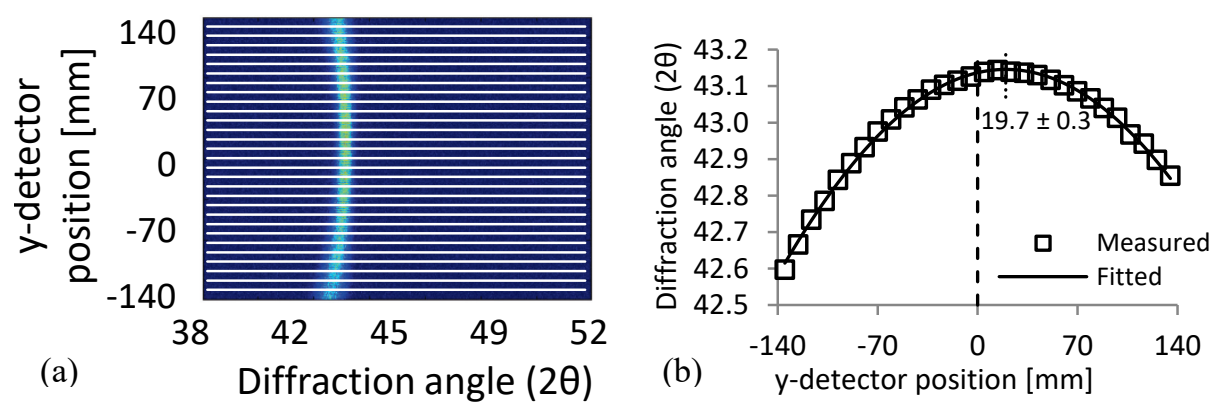
Fig. 9: (a) Diffraction
cone of Mo (110) plane
intercepting the
detector and (b) a
Gaussian fit over the
diffraction cone
showing the center
position.

By changing the monochromator tilt angle, the cone direction can be adjusted as the vertical incident angle onto the sample is changed. When the tilt angle is changed, the monochromator vertical offset (z-axis position) should also be adjusted to ensure that the beam is not restricted by the monochromator collimator. An iterative process therefore follows for the tilt and $z$-axis alignment.

\section{Detector alignment}

The first step in aligning the detector is to establish the relationship between the detector bins (chosen as $421 \times 421)$ and the detector active area. We know from the detector specification that the active area is $280 \times 280 \mathrm{~mm}^{2}$, therefore each bin represents an area of $0.67 \times 0.67 \mathrm{~mm}^{2}$.

In order to calculate the sample-to-detector distance, it is necessary to determine the total detector angular span. A mild steel calibration pin was mounted at the CoR and the detector positioned with the detector $2 \theta_{\mathrm{S}}$ angle (stth motor) at $94^{\circ}$ in order for the Fe (211) diffraction peak to be observed at the left edge of the detector. The detector was step-scanned in $4 \times 2^{\circ}$ steps to $2 \theta_{\mathrm{S}}=86^{\circ}$ and each peak were fit with a Gaussian function to determine the $x$-detector position of the peak center. The linear relationship between the observed peak positions as a function of $2 \theta_{\mathrm{S}}$ angle was used to calculate the total detector angular span as $13.9^{\circ}$. The sample to detector distance was established as $1148.5 \mathrm{~mm}$ by using the angular span and detector active length.

$\mathrm{Al}_{2} \mathrm{O}_{3}$ NIST standard powder was positioned on the sample table and the detector $2 \theta_{\mathrm{S}}$ angle step-scanned in 6 steps from $35^{\circ}$ to $100^{\circ}$. After stitching the acquired frames, Rietveld refinement was applied to the resulting diffraction pattern and the detector offset and neutron wavelength was determined as $-1.78^{\circ}$ and $1.646 \AA$ respectively. Using this wavelength, the monochromator exit port angle $\left(2 \theta_{\mathrm{M}}\right)$ was calculated as $82.7^{\circ}$, instead of the estimated $83.5^{\circ}$.

\section{Instrument gauge volume}

The vertical center of the beam was determined by $z$-step scanning (using the sz motor) a horizontal positioned mild steel bar through the primary beam with the apertures removed. The neutron counts from the Fe (211) diffraction peak were integrated and an analytical solution was fit to the measured data as a function of sz motor position. The center position was thereby resolved to within $0.2 \mathrm{~mm}$. The height of the lasers, theodolites and apertures were then adjusted to coincide with the bar center.

The two aperture horizontal positions were aligned by step scanning the slit horizontal offset axes (psho and ssho motors) respectively whilst recording the Fe (211) peak from a calibration cell positioned on the CoR. This measurement was done with each aperture at two different 
distances from the sample to calculate the mounting arm rotation required to ensure that the aperture always traverse parallel to the beam.

MPISI's beam divergence was subsequently measured by scattering from a mild steel bar using a nominal gauge volume of $2 \times 20 \times 2 \mathrm{~mm}^{3}$. Vertical and horizontal divergence were calculated by step-scanning the $z$ and $y$ sample table axes respectively as a function of primary slit position and fitting appropriate entry curve analytical functions to the integrated diffraction peak intensities. A horizontal divergence of $0.166^{\circ}$ and vertical divergence of $0.458^{\circ}$ were established.

\section{Summary}

Complete diffraction instrument alignment requires a systematic approach for alignment of individual components to establish a fixed instrument reference point and to optimize and characterize the incident (primary) and diffracted neutron beams. A number of these alignment steps are only performed during instrument commissioning eliminating the need for automation. There is however a number of routinely used procedures which can be automated in order to reduce human error and reduce the time needed to perform the alignment steps. The alignment and calibration procedures implemented at MPISI may also apply to other neutron diffraction instruments, depending on their specific configurations.

\section{References}

[1] A.M. Venter, P.R. van Heerden, D. Marais and J.C. Raaths, MPISI: The neutron strain scanner Materials Probe for Internal Strain Investigations at the SAFARI-1 research reactor, Physica B, In press. https://doi.org/10.1016/j.physb.2017.12.011

[2] G.A. Webster and R.W. Wimpory, (Eds.), Polycrystalline materials determination of residual stresses by neutron diffraction. Geneva: ISO, Technology Trends Assessment. (ISO/TTA3), 2002, pp. 29-37.

[3] Sylvac. Instructions for use - Dial Gauge S229. www.sylvac.ch Date of access: 14 May 2013 (2005)

[4] S. Flemming, Development of a distributed client server of system for the fully automatic instrument adjustment of a neutron diffractometer for residual stress analysis, Technische Fachhochschule Berlin, Berlin, 2012.

[5] H. Heer, M. Könnecke and D. Maden, The SINQ instrument control software system, Physica B 241-243 (1998) 124-126. https://doi.org/10.1016/S0921-4526(97)00528-0

[6] P.C. Brand and H.J. Prask, New methods for the alignment of instrumentation for residualstress measurements by means of neutron diffraction, J. Appl. Cryst. 27 (1994) 164-176. https://doi.org/10.1107/S0021889893007605

[7] A. Kelly and K.M. Knowles, Crystallography and Crystal Defects. 2nd ed. West Sussex, UK: John Wiley \& Sons, Ltd. 2012. https://doi.org/10.1002/9781119961468

[8] M.W. Johnson and M.R. Daymond, An optimum design for a time-of-flight neutron diffractometer for measuring engineering stresses, J. Appl. Cryst. 35 (2001) 49-57. https://doi.org/10.1107/S002188980101891X 